\title{
Aspectos respiratorios y de fisioterapia pulmonar en el niño con mucopolisacaridosis
}

\author{
Israel D. Cruz-Anleu*, Marvin V. Solís-Trujeque y Beth Sainz De La Peña-Hernández \\ Servicio Clínico de Neumología Pediátrica, Centro Regional de Alta Especialidad, Hospital de Especialidades Pediátricas Tuxtla Gutiérrez, Tuxtla \\ Gutiérrez, Chiapas, México
}

\begin{abstract}
Resumen
La mucopolisacaridosis es un grupo heterogéneo de enfermedades que se caracterizan por la acumulación lisosomal de sustancias intermedias del metabolismo de los mucopolisacáridos o glucosaminoglucanos. El trastorno respiratorio que caracteriza a los pacientes con mucopolisacaridosis es la enfermedad pulmonar restrictiva crónica, por lo que la rehabilitación pulmonar, cuyo objetivo es mejorar los síntomas respiratorios, y la fisioterapia respiratoria, mejorarán la ventilación pulmonar y la biomecánica respiratoria deteriorada. Es necesario el seguimiento por el neumólogo infantil, quien cuantificará la función pulmonar y vigilará los síntomas de obstrucción nocturna y de restricción pulmonar con ayuda de estudios como la espirometría, la pletismografía y la prueba de la caminata de 6 minutos, por mencionar algunas. También es muy importante realizar un programa individualizado de técnicas de fisioterapia respiratoria y de ejercicios. Todo lo anterior, con el objetivo de evaluar la función pulmonar como un marcador de respuesta al uso de cualquiera de las terapias indicadas en la mucopolisacaridosis.
\end{abstract}

Palabras clave: Mucopolisacaridosis. Fisioterapia respiratoria. Función pulmonar.

\section{Respiratory aspects and pulmonary physiotherapy in the child with mucopolysaccharidosis}

\begin{abstract}
Mucopolysaccharidosis is a heterogeneous group of diseases characterized by the lysosomal accumulation of intermediates in the metabolism of mucopolysaccharides or glycosaminoglycans. The respiratory disorder that characterizes patients with mucopolysaccharidosis is the chronic restrictive lung disease. Therefore, pulmonary rehabilitation aimed at improving respiratory symptoms and respiratory physiotherapy will improve pulmonary ventilation and impaired respiratory biomechanics in patients with mucopolysaccharidosis. Follow-up by the pediatric pulmonologist is necessary to quantify lung function and monitor the symptoms of nocturnal obstruction and pulmonary restriction with the help of studies such as spirometry, plethysmography and the 6-minute walk test, among others. It is also very important to perform an individualized program of respiratory physiotherapy techniques and exercises. Overall, all of these steps are followed for evaluating lung function as a marker of response to the use of any of the therapies indicated in mucopolysaccharidosis.
\end{abstract}

Keywords: Mucopolysaccharidosis. Pulmonary function. Pulmonary physiotherapy.

\section{Correspondencia:}

*Israel D. Cruz-Anleu

E-mail: canleu2@yahoo.com.mx
Fecha de recepción: 28-08-2020

Fecha de aceptación: 10-11-2020

DOI: 10.24875/BMHIM.20000266
Disponible en internet: 23-07-2021

Bol Med Hosp Infant Mex. 2021;78(4):318-325

www.bmhim.com 1665-1146/@ 2020 Hospital Infantil de México Federico Gómez. Publicado por Permanyer. Este es un artículo open access bajo la licencia CC BY-NC-ND (http://creativecommons.org/licenses/by-nc-nd/4.0/). 


\section{Introducción}

La mucopolisacaridosis (MPS) es un grupo heterogéneo de enfermedades que se caracterizan por la acumulación lisosomal de sustancias intermedias del metabolismo de los mucopolisacáridos o glucosaminoglucanos, macromoléculas que proporcionan soporte estructural a la matriz extracelular ${ }^{1}$. La presentación clínica, la edad del diagnóstico, el tratamiento y sus complicaciones varían dependiendo del tipo de MPS ${ }^{1}$. El tratamiento es variable, pero el objetivo final es proveer una cantidad adecuada de enzima, evitando o corrigiendo la acumulación de glucosaminoglucanos ${ }^{1,2}$. El trastorno respiratorio que caracteriza a los pacientes con MPS es la enfermedad pulmonar restrictiva crónica, por lo que la rehabilitación pulmonar, cuyo objetivo es mejorar los síntomas respiratorios por medio de sus tres componentes (educación, fisioterapia y ejercicio), puede ser una opción en la búsqueda de un tratamiento que mejore los síntomas respiratorios restrictivos que se presentan, liberando de secreciones la vía aérea y mejorando la calidad de vida de los pacientes con MPS ${ }^{3,4}$.

El objetivo de este artículo fue describir las características respiratorias de la MPS y los tratamientos de fisioterapia respiratoria que pueden utilizarse en los niños con MPS. Se realizó una revisión narrativa de artículos en inglés, español y francés en PubMed, Scielo, Latindex, Google académico y EBSCOhost. No hubo limitaciones en cuanto a fecha de publicación ni diseño metodológico, siempre y cuando describieran un aspecto relevante del tema. La revisión de artículos se realizó durante un periodo de 3 meses (1 de marzo a 30 de junio de 2020), considerando aquellos que incluían los siguientes términos de búsqueda (MeSH): mucopolysaccharidosis, physiotherapy techniques, respiratory therapies, pulmonary function test y pulmonary rehabilitation. Además, se examinó la lista de referencias de los artículos identificados en busca de artículos potencialmente relevantes, los cuales se consideraron para su inclusión siempre y cuando describieran algún aspecto importante de los pacientes con mucopolisacáridos y los trastornos respiratorios que les afectan.

\section{Epidemiología}

La MPS es una enfermedad infrecuente y variable, dependiendo de los rasgos étnicos y geográficos ${ }^{5-7}$. En México, las guías del Centro Nacional de Excelencia Tecnológica en Salud refieren una prevalencia de MPS
I de 0.69-3.8/100,000 recién nacidos vivos, y de MPS II de $1 / 132,000$ varones recién nacidos vivos ${ }^{8,9}$. Durante 2019, en el Hospital de Especialidades Pediátricas de Chiapas se encontraban bajo seguimiento 3285 niños, de los cuales 36 se diagnosticaron con MPS, lo que correspondió al $1 \%$ del total de niños consultados. La media de edad fue de 9.8 años, la relación masculino/ femenino fue de 1:1 (18 casos por sexo) y el grupo con mayor frecuencia de casos fue el de 11 a 15 años de edad (Fig. 1). En cuanto a los límites por edad, se observó una edad mínima de 1 año (un caso) y una máxima de 18 años (un caso). La MPS que más predominó fue la de tipo I (63.8\%), el síntoma más común fue el ronquido nocturno (69.4\%) y las patologías de mayor asociación fueron la rinosinusitis crónica (44.4\%) y el asma (27.7\%) (Tabla 1).

\section{Clasificación de la mucopolisacaridosis}

La clasificación de la MPS es fundamental para iniciar un diagnóstico y un tratamiento oportuno. La MPS se divide en varios tipos ${ }^{1,2}$ :

- MPS I o síndrome de Hurler (Hurler, Hurler-Scheie y Scheie).

- MPS II o síndrome de Hunter (variantes A y B).

- MPS III o síndrome de Sanfilippo (variantes A-D).

- MPS IV o síndrome de Morquio (variantes A y B).

- MPS VI o síndrome de Maroteaux-Lamy.

- MPS VII o síndrome de Sly.

- MPS IX o síndrome de Natowicz.

Se debe considerar que cada fenotipo presenta diferentes alteraciones anatomopatológicas en el sistema respiratorio, lo que resulta de suma importancia dada la necesidad de un tratamiento de reemplazo enzimático, el manejo multidisciplinario y la corrección de los síntomas respiratorios antes de que se presenten complicaciones graves ${ }^{1,10}$.

\section{Características anatomofisiopatológicas de los pacientes con mucopolisacaridosis}

A pesar de la diversidad del espectro clínico, se pueden observar infecciones respiratorias recurrentes y obstrucción de la vía aérea superior por hipertrofia adenoamigdalina y macroglosia, engrosamiento de la mucosa traqueobronquial y tórax restrictivo que puede llevar a presentar padecimientos respiratorios cróni$\cos ^{1,11,12}$. En la tabla 2 se resumen las características anatomopatológicas respiratorias de las diversas MPS. 
Tabla 1. Características demográficas y respiratorias de los pacientes con mucopolisacaridosis atendidos en el Hospital de Especialidades Pediátricas de Chiapas $(\mathrm{n}=36)$

\begin{tabular}{|l|c|}
\hline Características & Media \pm DE \\
\hline Edad (años) & $9.8 \pm 4.3$ \\
\hline Peso (kg) & $17.9 \pm 7.5$ \\
\hline Talla (cm) & $100.9 \pm 17.8$ \\
\hline Sexo (F) & $\mathbf{n}(\%)$ \\
\hline MPS I & $18(50)$ \\
\hline MPS II & $23(63.8)$ \\
\hline MPS IV & $5(13.8)$ \\
\hline MPS VI & $5(13.8)$ \\
\hline Rinosinusitis crónica & $3(8.3)$ \\
\hline SAHOS & $16(44.4)$ \\
\hline Hipercapnia nocturna & $4(11.1)$ \\
\hline Hipoxemia & $4(11.1)$ \\
\hline Ronquido nocturno & $5(13.8)$ \\
\hline Asma & $25(69.4)$ \\
\hline & $10(27.7)$ \\
\hline
\end{tabular}

DE: desviación estándar; F: femenino; MPS: mucopolisacaridosis; SAHOS: apneas o hipopneas obstructivas del sueño.

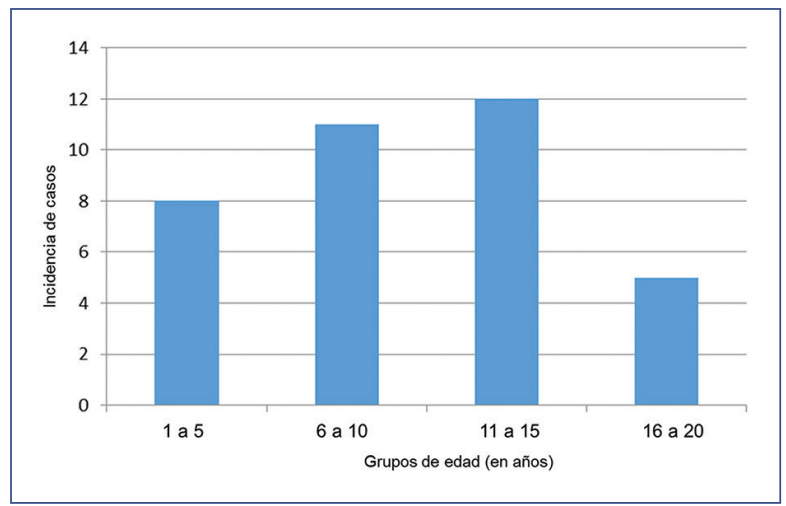

Figura 1. Incidencia de casos de mucopolisacaridosis bajo seguimiento en el Hospital de Especialidades Pediátricas, Tuxtla Gutiérrez, Chiapas, en menores de 20 años.

Las manifestaciones otorrinolaringológicas en los pacientes con MPS generalmente son rinorrea nasal crónica o persistente y secreciones de la vía aérea inferior. Estas son secundarias a alguna obstrucción
Tabla 2. Características anatomopatológicas del aparato respiratorio en los pacientes con mucopolisacaridosis y enzima deficiente

\begin{tabular}{|c|c|c|}
\hline MPS & Enzima deficiente & $\begin{array}{l}\text { Alteraciones del aparato } \\
\text { respiratorio características }\end{array}$ \\
\hline I & I-iduronidasa & $\begin{array}{l}\text { Obstrucción de la vía aérea } \\
\text { superior } \\
\text { Micrognatia } \\
\text { Enfermedad pulmonar } \\
\text { restrictiva }\end{array}$ \\
\hline II & Iduronato 2 sulfatasa & $\begin{array}{l}\text { Macroglosia } \\
\text { Hipertrofia gingival } \\
\text { Cuello y tórax cortos } \\
\text { Alteraciones del macizo } \\
\text { facial }\end{array}$ \\
\hline III & $\begin{array}{l}\text { Heparán N sulfatasa } \\
\text { Alfa N } \\
\text { acetilglucosaminidasa } \\
\text { Alfa-glucosamida } \\
\text { acetiltransferasa } \\
\text { N-acetilglucosamina- } \\
\text { 6-sulfatasa }\end{array}$ & $\begin{array}{l}\text { Labio inferior grueso y } \\
\text { evertido } \\
\text { Surco nasolabial prominente } \\
\text { Hipoacusia } \\
\text { Alteraciones en la deglución }\end{array}$ \\
\hline IV & $\begin{array}{l}\text { Galactosa 6-sulfatasa } \\
\text { Beta-galactosidasa }\end{array}$ & $\begin{array}{l}\text { Displasia esquelética } \\
\text { Talla baja } \\
\text { Tórax restrictivo: } \\
\text { Pectus carinatum } \\
\text { Tórax en forma de remo } \\
\text { Cifosis } \\
\text { Escoliosis y cifoescoliosis }\end{array}$ \\
\hline VI & Arilsulfatasa B & $\begin{array}{l}\text { Afectación esquelética } \\
\text { torácica } \\
\text { Macrocefalia } \\
\text { Puente nasal deprimido } \\
\text { Restricción pulmonar }\end{array}$ \\
\hline VII & Beta-glucuronidasa & $\begin{array}{l}\text { Macrocefalia } \\
\text { Alteraciones del macizo } \\
\text { facial } \\
\text { Cuello corto }\end{array}$ \\
\hline IX & Hialuronidasa & $\begin{array}{l}\text { Paladar hendido } \\
\text { Puente nasal deprimido } \\
\text { Aplanamiento facial } \\
\text { Pérdida de la audición } \\
\text { Disostosis múltiple }\end{array}$ \\
\hline
\end{tabular}

MPS: mucopolisacaridosis.

mecánica, a un proceso inflamatorio persistente y a la calidad de la secreción producida por las glándulas de la mucosa y submucosa, donde la hipersecreción es producto del déficit de dermatán sulfato y de queratán sulfato $^{13}$. Esta infiltración de glucosaminoglucanos se manifiesta también en otras estructuras de la vía aérea superior, como la laringe, las adenoides y las amígdalas palatinas, produciendo hipotonía y mayor colapso de la vía aérea, y manifestando apneas o hipopneas obstructivas del sueño en los niños con MPS ${ }^{14}$. En 
cuanto al parénquima, los niños con MPS cursan con inflamación importante y disminución del lumen de la vía aérea, provocando una irregularidad de sus paredes, lo que produce una hipersecreción bronquial y una disminución del drenaje mucociliar que resulta en broncoconstricción y broncorrea, las cuales pueden complicar los procedimientos de inducción anestésica ${ }^{15}$. Respecto a las alteraciones del fuelle torácico, estas se manifiestan en aquellas MPS con compromiso esquelético más grave (tipos I, II, IV y VI) y se caracterizan por deformidades y rigidez de las articulaciones costales, horizontalización de las costillas, escoliosis, cifosis, xifoescoliosis y pectus carinatum. También la hepatoesplenomegalia grave se asocia con estas malformaciones, provocando una mayor reducción de la capacidad vital ${ }^{16,17}$ y manifestando una enfermedad pulmonar restrictiva de diversos grados ${ }^{18}$.

\section{Valoración respiratoria del paciente con mucopolisacaridosis}

La evaluación respiratoria del niño con MPS debe centrarse en realizar un adecuado interrogatorio médico, con la búsqueda intencionada de síntomas sugerentes de hipercapnia restrictiva ${ }^{19}$, como temblores finos (flapping), hipotensión arterial (por vasodilatación periférica), somnolencia diurna, alteraciones del ritmo vigilia-sueño, cefalea, náusea y vómito. En casos extremos se manifestarán síntomas y signos de coma hipercápnico (desorientación, sudoración, obnubilación y dificultad respiratoria) ${ }^{20}$. La gasometría arterial y la monitorización de la saturación periférica de oxígeno con oximetría de pulso serán de gran ayuda para descartar hipoventilación alveolar crónica e hipoxemia ${ }^{21,22}$. Los valores anormalmente altos de bicarbonato y el exceso de base $>4 \mathrm{mEq} / \mathrm{L}$ en la gasometría arterial apoyan el diagnóstico de anomalías del intercambio gaseoso durante el sueño o la vigilia en los pacientes con MPS e hipercapnia crónica ${ }^{20,21}$.

Las pruebas de función respiratoria, como la espirometría, la pletismografía, la difusión de monóxido de carbono, la caminata de 6 minutos y la medición de la fuerza muscular inspiratoria-espiratoria máxima, deben realizarse sistemáticamente ${ }^{23}$. La mayor limitación para realizar este tipo de pruebas es la necesidad de la máxima disponibilidad posible por parte del paciente ${ }^{23,24}$. La pletismografía determina la capacidad pulmonar total y sugiere, por medio del índice volumen residual/ capacidad pulmonar total, la presencia de una enfermedad restrictiva pulmonar. La espirometría apoya el diagnóstico de una enfermedad obstructiva y sugiere restricción pulmonar con ayuda de una capacidad vital forzada (FVC) $<70 \%$ y un volumen espirado forzado durante el primer segundo/capacidad vital forzada (FEV1/FVC) igual o superior al límite inferior de la normalidad. En los pacientes con debilidad muscular respiratoria, los valores de FVC $<1-1.5$ L o FEV1 $<40 \%$ del predicho sugerirán la presencia de hipercapnia nocturna ${ }^{17,24-27}$. Las presiones máximas permiten evaluar y cuantificar, en forma global, la fuerza de los músculos respiratorios al realizar una maniobra inspiratoria $\left(\mathrm{Pi}_{\max }\right)$ o espiratoria $\left(\mathrm{Pe}_{\max }\right)$ forzada en contra de una vía aérea ocluida. Son útiles en pacientes con trastornos neuromusculares y patologías restrictivas pulmonares ${ }^{28,29}$. Un valor de $\mathrm{Pi}_{\max }<60 \mathrm{cmH}_{2} \mathrm{O}$ es un signo de debilidad de los músculos respiratorios y de hipoventilación nocturna; un valor de $\mathrm{Pi}_{\max }<30 \mathrm{cmH}_{2} \mathrm{O}$ es indicativo de insuficiencia respiratoria hipercápnica en vigilia ${ }^{30}$. La caminata de 6 minutos evalúa la capacidad de ejercicio de los pacientes con patología respiratoria; es adecuada para evaluar los efectos de la rehabilitación y se utiliza con fines pronósticos. Sus ventajas son su alta reproducibilidad (coeficiente de variabilidad del $8 \%$ ), ser fácil de realizar, ser bien tolerada y reflejar mejor las actividades de la vida diaria que otras pruebas ${ }^{28}$. La caminata de shuttle y la prueba 1-min sit to stand son también de gran ayuda para valorar la capacidad aeróbica de los pacientes con MPS y patología pulmonar crónica ${ }^{31-33}$. Los estudios de imagen, como la telerradiografía de tórax y la tomografía computada (TC) con reconstrucción, corroborarán la presencia de alteraciones musculoesqueléticas torácicas. Es recomendable realizar conjuntamente una videobroncoscopia y una TC en fase inspiratoria-espiratoria para evaluar la presencia de colapso dinámico de la vía aérea (traqueobroncomalacia) ${ }^{25,26,34}$. Otros estudios, como la serie esofagogastroduodenal con mecánica de la deglución, la videofluoroscopia, el salivograma, la gammagrafía con búsqueda de broncoaspiración tardía y el estudio fibroendoscópico de la deglución, se deben considerar para descartar reflujo gastroesofágico y alteraciones de la deglución en los pacientes con MPS y manifestaciones respiratorias crónicas $^{35-41}$. También son importantes pruebas como la electrocardiografía y los estudios que evalúan el compromiso funcional otorrinolaringológico y auditivo, como la impedanciometría, la audiometría, los potenciales evocados y la nasofibroscopía ${ }^{17}$. Es muy importante descartar un trastorno del sueño por medio de una polisomnografía con titulación de un sistema de apoyo respiratorio con presión positiva; esto debido a que los pacientes con MPS y deterioro sumamente 
significativo de la función pulmonar presentan una alta asociación con la presencia de trastornos en la arquitectura del sueño, apneas y alteraciones del intercambio gaseoso arteriovenoso ${ }^{23}$. El dióxido de carbono exhalado $>45 \mathrm{mmHg}$ es indicativo de hipoventilación alveolar ${ }^{21}$.

\section{Técnicas de terapia física y ejercicio}

El niño con MPS presenta una reducción en la tolerancia al ejercicio y una limitación en los arcos de movilidad de las extremidades ${ }^{8,9}$. Estos problemas limitan la movilidad y afectan la calidad de vida, por lo que se recomienda la práctica deportiva diaria adaptada a cada individuo, dados los beneficios que se obtienen desde el punto de vista físico, psíquico y de desarrollo personal y social ${ }^{42}$. Algunas técnicas de terapia física utilizadas en los pacientes con MPS se basan en estiramientos para reducir las contracciones musculares, aumentar la amplitud articular y fortalecer la musculatura con el fin de mejorar la marcha y el equilibrio. También es necesaria la disociación de la cintura escapular y pélvica por medio de ejercicios que ayuden a mejorar la coordinación motora y la propiocepción, baile: saltos y juegos que mejoren el movimiento fino. Se deberá evitar la hiperextensión de la cabeza por causa del compromiso de la articulación atlantoaxial $\left.\right|^{8,9,43}$.

\section{Técnicas de fisioterapia respiratoria}

De acuerdo con la fisiopatología de la enfermedad pulmonar restrictiva que sufren los pacientes con MPS, la rehabilitación pulmonar es la opción más adecuada en niños y adultos con MPS y enfermedades pulmonares crónicas que presentan una disminución de las actividades de la vida diaria ${ }^{3,44,45}$. Los objetivos de la rehabilitación pulmonar son reducir los síntomas, optimizar el estado funcional y reducir los gastos en salud $^{3}$. La fisioterapia respiratoria mejorará la ventilación pulmonar y la biomecánica respiratoria deteriorada en los pacientes con MPS, gracias a la ayuda de programas personalizados que evalúan la tolerancia al ejercicio muscular periférico y respiratorio de forma conjunta ${ }^{45}$.

El objetivo de la fisioterapia respiratoria es mejorar la alteración restrictiva pulmonar en la MPS, la cual es el resultado de la obstrucción provocada en la vía aérea superior, el acortamiento muscular respiratorio, la deformidad del tórax y de la columna dorsal, la hepatomegalia y la broncoaspiración causada por la disfagia ${ }^{41}$. Estos mecanismos llevarán a una reducción en la expansión y la movilidad torácica, y a la producción de tos ineficaz que se traducirá en hipersecreción, infecciones recurrentes y apneas del sueño $0^{16-18,43}$. Las guías de práctica clínica para el cuidado respiratorio de los pacientes con MPS o enfermedad de Pompe recomiendan iniciar los cuidados respiratorios tempranamente y realizarlos de una a cuatro veces al día ${ }^{46}$. El objetivo de las maniobras indicadas es mejorar la higiene bronquial. Entre estas maniobras se encuentran las vibraciones, la percusión (clapping), el drenaje postural (evitar la posición de Trendelemburg), la tos asistida mediante la maniobra de aceleración del flujo espiratorio y la asistencia de la tos con una bolsa de reanimación manual. Siempre deberán indicarse junto con maniobras dinámicas de estiramiento, fortalecimiento, masajes y posicionamiento adecuado ${ }^{46}$. También pueden utilizarse nebulizaciones y limpieza de la vía aérea superior con solución fisiológica ${ }^{43}$. Existen otras técnicas muy efectivas para liberar las secreciones de la vía aérea superior y que pueden aplicarse en niños cooperadores con obstrucción nasal importante, como son la desobstrucción retrofaríngea retrógrada, la tos nasal y las duchas nasales asociadas a tos provocada ${ }^{47}$.

La tos es uno de los mecanismos de defensa del árbol traqueobronquial, cuyo objetivo es expulsar secreciones de manera espontánea, provocada o dirigida. En los niños con MPS, la tos puede estar disminuida, por lo que las técnicas que ayudan a mejorar su efectividad, sobre todo cuando existe un pico flujo de tos (PFT) < $270 \mathrm{~L} / \mathrm{min}$, son las siguientes: tos provocada, técnica que se da en un acto reflejo desencadenado tras una estimulación manual en los centros tusígenos de la tráquea ${ }^{47,48}$; y asistencia manual de la tos, que se basa en el aumento de la capacidad inspiratoria seguida de un incremento del esfuerzo espiratorio a través de una maniobra expulsiva (compresión abdominal) con las manos del terapeuta ${ }^{49}$. Otras técnicas buscan obtener un volumen espiratorio mayor, con el objetivo de mejorar la desinsuflación pulmonar al evitar la aparición de una zona de estrechamiento bronquial (espiración lenta prolongada $)^{50,51}$. También pueden aplicarse técnicas como la espiración lenta total con glotis abierta, técnica pasiva o activa-asistida que se realiza a partir de la capacidad residual funcional y que continúa hasta el volumen residual. Siempre debe situarse la región a permeabilizar en infralateral; de esta forma, se asiste la desinsuflación y se favorece la eliminación de secreciones del árbol bronquial medio y distal ${ }^{51}$. Las técnicas respiratorias instrumentadas están indicadas en 
Tabla 3. Resumen de las técnicas de fisioterapia pulmonar instrumentadas que pueden utilizarse en los pacientes con mucopolisacaridosis

\begin{tabular}{|c|c|}
\hline Tipo de fisioterapia instrumentada & Técnica y objetivo \\
\hline $\begin{array}{l}\text { Máquina de tos asistida } \\
\text { (insuflador-exuflador mecánico) }\end{array}$ & $\begin{array}{l}\text { Técnica: comienza con una inspiración máxima (presión positiva que oscila entre }+30 \text { y } \\
+50 \mathrm{cmH}_{2} \mathrm{O} \text { ) combinada con presión negativa (presión de exuflación que oscila entre } \\
-20 \text { y }-40 \mathrm{cmH}_{2} \mathrm{O} \text { ). El flujo pico de tos puede aumentar del } 14-100 \% \text {. } \\
\text { Indicaciones: casos de obstrucción bronquial proximal, debilidad muscular, tapones de } \\
\text { moco en atelectasias, neumonía, bronquiolitis. }\end{array}$ \\
\hline $\begin{array}{l}\text { Presión espiratoria positiva (TheraPEP }{ }^{\circledR} \text {, } \\
\text { Threshold Peep, EZPAP }{ }^{\circledR} \text { ) }\end{array}$ & $\begin{array}{l}\text { Técnica: el objetivo es modular el flujo espiratorio para evitar el colapso precoz de la } \\
\text { vía aérea y favorecer el drenaje de secreciones. } \\
\text { Objetivo: mejorar el drenaje de las secreciones al incrementar la presión del gas } \\
\text { detrás del moco mediante ventilación colateral o previniendo el colapso de la vía } \\
\text { aérea durante la espiración. }\end{array}$ \\
\hline Ventilación percusiva intrapulmonar & $\begin{array}{l}\text { Técnica: el mecanismo de acción consiste en liberar corrientes de gas con flujos altos } \\
\text { en la vía aérea a una frecuencia de } 100-300 \text { impulsos por minuto controlados mediante } \\
\text { un interruptor llamado Phasitron y a baja presión. } \\
\text { Objetivos: producir broncodilatación por aumento de la presión y humidificación de la } \\
\text { vía aérea. Desobstruye el árbol bronquial periférico. }\end{array}$ \\
\hline $\begin{array}{l}\text { Oscilación a alta frecuencia en la pared } \\
\text { torácica (chaleco oscilador) }\end{array}$ & $\begin{array}{l}\text { Técnica: se realiza con un chaleco neumático que aplica pulsos de aire a presión } \\
\text { positiva y vibración externa en la pared torácica por medio de cambios oscilantes } \\
\text { bruscos en la presión del chaleco. } \\
\text { Objetivo: el mecanismo de acción es inflar y desinflar gentil y rápidamente, para } \\
\text { comprimir y liberar la pared del tórax a una frecuencia de entre } 5 \text { y } 20 \text { ciclos por } \\
\text { segundo, con el propósito de crear un flujo de aire en el interior de la vía aérea. } \\
\text { Permite despegar el moco para ser movilizado hacia la vía aérea central, de modo que } \\
\text { pueda ser expulsado por la tos o la succión. }\end{array}$ \\
\hline
\end{tabular}

pacientes con volúmenes pulmonares altamente reducidos y tos ineficaz (PFT < $160 \mathrm{~L} / \mathrm{min})$ secundaria a malformaciones como cifoescoliosis, debilidad muscular respiratoria y restricción pulmonar ${ }^{48}$. Las más utilizadas son la máquina de tos asistida ${ }^{52-54}$, la ventilación percusiva intrapulmonar ${ }^{55-57}$, el percutor acústico (Vibralung $\left.^{\circledR}\right)^{58,59}$ y el chaleco oscilador $\left(\text { Vest }^{\circledR}\right)^{47,53,60-62}$. Existen otros instrumentos que aumentan la fuerza muscular respiratoria, como Threshold IMT y PEEP63, TheraPEP ${ }^{\circledR 47}$ y EZPAP ${ }^{\circledR 64,65}$. En la tabla 3 se resumen las técnicas instrumentadas mencionadas.

La ventilación mecánica no invasiva es otro tratamiento efectivo en las enfermedades restrictivas asociadas a apneas del sueño graves e hipoventilación. Entre sus beneficios se encuentran la mejora de la permeabilidad de la vía aérea superior, el restablecimiento de la sensibilidad de los quimiorreceptores centrales, la mejora de la calidad del sueño y de la distensibilidad pulmonar, y el enlentecimiento de la deformidad de la caja torácica ${ }^{66,67}$.

\section{El papel del especialista pediátrico}

Las manifestaciones pulmonares son diversas y se manifiestan más precozmente en las MPS de los tipos I, II y VI. La MPS de tipo IV presentará con mayor frecuencia deformidades torácicas graves y se complicarán con compresiones extrínsecas de la vía aérea ${ }^{1,2}$. La MPS de tipo III se asociará a hipersecreción bronquial, defectos del drenaje mucociliar, trastornos de la deglución y aspiración pulmonar crónica ${ }^{1,2,17,41}$, lo que hace necesario el seguimiento por neumología infantil para cuantificar la función pulmonar y vigilar los síntomas de obstrucción nocturna y restricción pulmonar. En los pacientes con MPS que cooperen es necesaria la evaluación de la función pulmonar dos veces al año, sobre todo en aquellos que inician alguna terapia de reemplazo enzimático o trasplante de médula ósea ${ }^{8,9,17}$. Las pruebas obligatorias de función respiratoria son la caminata de 6 minutos, la pletismografía y la espirometría, para clasificar tanto la obstrucción como la restricción pulmonar y la calidad de vida ${ }^{17}$. También es importante la valoración por el neumólogo-rehabilitador pulmonar para la evaluación de un programa individualizado de fisioterapia respiratoria y de ejercicios. Todo esto con el objetivo de evaluar la función pulmonar como un marcador de respuesta al uso de cualquiera de las terapias indicadas en la MPS $8,9,18$.

\section{Responsabilidades éticas}

Protección de personas y animales. Los autores declaran que para esta investigación no se han realizado experimentos en seres humanos ni en animales. 
Confidencialidad de los datos. Los autores declaran que han seguido los protocolos de su centro de trabajo sobre la publicación de datos de pacientes.

Derecho a la privacidad y consentimiento informado. Los autores declaran que en este artículo no aparecen datos de pacientes.

\section{Conflicto de intereses}

Los autores declaran no tener ningún conflicto de intereses.

\section{Financiamiento}

Ninguno.

\section{Bibliografía}

1. Suárez-Guerrero JL, Gómez-Higuera PJI, Arias-Flores JS, Contreras-García GA. Mucopolisacaridosis: características clínicas, diagnóstico y de manejo. Rev Chil Pediatr. 2016:87:295-304.

2. Amartino H, Barreiro C, Cozzo V, Czornyj L, Drelichman G, Eiroa H, et al Consenso de diagnóstico y tratamiento de la mucopolisacaridosis de tipo 1. Arch Argent Pediatr. 2008;106:361-8.

3. Spruit MA, Singh SJ, Garvey C, Zuwallack R, Nici L, Rochester C, et al. An official American Thoracic Society/European Respiratory Society statement: key concepts and advances in pulmonary rehabilitation. Am J Respir Crit Care Med. 2013:188:e13-64.

4. Bott J, Blumenthal S, Buxton M, Ellum S, Falconer C, Garrod R, et al. Guidelines for the physiotherapy management of the adult, medical spontaneously breathing patient. Thorax. 2009;64:i1-51.

5. Lin HY, Lin SP, Chuang CK, Niu DM, Chen MR, Tsai FJ, et al. Incidence of the mucopolysaccharidoses in Taiwan, 1984-2004. Am J Med Genet A. 2009;149A:960-4.

6. Baehner F, Schmiedeskamp C, Krummenauer F, Miebach E, Bajbouj M Whybra $C$, et al. Cumulative incidence rates of the mucopolysaccharidoses in Germany. J Inherit Metab Dis. 2005;28:1011-7.

7. Del Toro-Riera M. [Follow-up of patients with Hunter syndrome: the Hunter Outcome Survey (HOS) registry]. Rev Neurol. 2007;44:S13-7.

8. Diagnóstico y tratamiento de la mucopolisacaridosis tipo I en edad pediátrica. Secretaría de Salud, Ciudad de México; 2017. Disponible en: http://www.cenetec.salud.gob.mx/contenidos/gpc/catalogoMaestroGPC.html

9. Diagnóstico y tratamiento de la mucopolisacaridosis tipo II en edad pediátrica. Secretaría de Salud, Ciudad de México; 2011. Disponible en www.cenetec.salud.gob.mx/interior/gpc.html

10. Pineda-Galindo LF, Moranchel-García L. Síndrome de Hurler-Scheie: mucopolisacaridosis tipo I. Med Int Mex. 2015;31:99-105.

11. Guelbert NB, Amartino H, Arberas CL, Azar NB, Bay ML, Fainboim A et al. Guía para el diagnóstico, seguimiento y tratamiento de la mucopolisacaridosis de tipo II (MPS-II) o enfermedad de Hunter. Arch Argent Pediatr. 2011:109:175-81.

12. Hernández-Sánchez BM, Peña-Olvera S. Mucopolisacaridosis: ¿un reto para el anestesiólogo? Rev Mex Anest. 2018;41:S162-5.

13. Wold SM, Derkay CS, Darrow DH, Proud V. Role of the pediatric otolaryngologist in diagnosis and management of children with mucopolysaccharidoses. Int J Pediatr Otorhinolaryngol. 2010;74:27-31.

14. Yeung $\mathrm{AH}$, Cowan MJ, Horn B, Rosbe KW. Airway management in children with mucopolysaccharidoses. Arch Otolaryngol Head Neck Surg. 2009; 135:73-9.

15. Walter R, Belani KG, Braunlin EA, Bruce IA, Hack H, Harmatz PR, et al. Anaesthesia and airway management in mucopolysaccharidosis. J Inherit Metab Dis. 2013;36:211-9.

16. Schmidt M, Breyer S, Löbel U, Yarar S, Stücker R, Ullrich K, et al. Musculoskeletal manifestations in mucopolysaccharidosis type I (Hurler syndrome) following hematopoietic stem cell transplantation. Orphanet $J$ Rare Dis. 2016;11:93.

17. Hernández J. Compromiso respiratorio en mucopolisacaridosis. Neumol Pediatr. 2013;8:27-33.

18. Broomfield A, Sims J, Mercer J, Hensman P, Ghosh A, Tylee K, et al. The evolution of pulmonary function in childhood onset mucopolysaccharidosis type I. Mol Genet Metab. 2021;132:94-9.
19. Basille $D$, Andréjak $C$, Jounieaux $V$. Insuficiencia respiratoria crónica: manejo por el médico general. EMC. 2016;20:1-9.

20. Diab-Cáceres L, Vázquez-Espinosa E, Marcos C, Gómez-Punter RM. Insuficiencia respiratoria crónica. Etiopatogenia. Clínica. Diagnóstico. Medicine. 2014;11:3735-41.

21. Cruz-Anleu ID, Baños-Mejía BO, Ovando-Fonseca JE. Aspectos básicos de la evaluación de la función pulmonar en el paciente con enfermedades neuromusculares. Rehabilitación (Madr). 2013;47:113-9.

22. Gutiérrez-Muñoz FR. Insuficiencia respiratoria aguda. Acta Med Per. 2010;27:286-97

23. Wooten WI $3^{\text {rd }}$, Muenzer J, Vaughn BV, Muhlebach MS. Relationship of sleep to pulmonary function in mucopolysaccharidosis II. J Pediatr. 2013;162:1210-5.

24. Kamin W. Diagnosis and management of respiratory involvement in Hunter syndrome. Acta Pædiatr. 2008:97:57-60.

25. Rutten M, Ciet P, Van den Biggelaar E, Oussoren E, Langendonk JG, Van der Ploeg AT, et al. Severe tracheal and bronchial collapse in adults with type II mucopolysaccharidosis. Orphanet J Rare Dis. 2016;11:50.

26. Murgu SD, Colt HG. Tracheobronchomalacia and excessive dynamic airway collapse. Clin Chest Med. 2006;11:388-406.

27. Martínez-Carrasco C, Villa-Asensi JR, Luna-Paredes MC, Osona-Rodríguez-de-Torres FB, Peña-Zarza JA, Larramona-Carrera $\mathrm{H}$, et al. Enfermedad neuromuscular: evaluación clínica y seguimiento desde el punto de vista neumológico. An Pediatr (Barc). 2014;81:258.e1-17.

28. Figueroa BMG, Trinidad-Mozó FM, Rodríguez DJC. Laboratorio de función pulmonar. Rev Med Clin Condes. 2015;26:376-86.

29. Mora-Romero UJ, Gochicoa-Rangel L, Guerrero-Zuñiga S, Cid-Juárez S, Silvia-Cerón M, Salas-Escamilla I, et al. Presiones inspiratoria y espiratoria máximas: recomendaciones y procedimiento. Neumol Cir Torax. 2014;73:247-53.

30. Prado F, Salinas-Flores PC, Zenteno D, Vera R, Flores O, García C, et al. Recomendaciones para los cuidados respiratorios del niño y adolescente con enfermedades neuromusculares. Neumol Pediatr. 2010:5:74-89.

31. Crook S, Büsching G, Schultz K, Lehbert N, Jelusic D, Keusch S, et al. A multicentre validation of the 1-min sit-to-stand test in patients with COPD. Eur Respir J. 2017;49:1601871.

32. Bohannon RW, Crouch R. 1-Minute sit-to-stand test: systematic review of procedures, performance, and clinimetric properties. J Cardiopulm Rehabil Prev. 2019;39:2-8.

33. Haley SM, Fragala Pinkham MA, Dumas HM, Ni P, Skrinar AM, Cox GF. A physical performance measure for individuals with mucopolysaccharidosis type I. Dev Med Child Neurol. 2006;48:576-81.

34. Morimoto N, Kitamura M, Kosuga M, Okuyama T. CT and endoscopic evaluation of larynx and trachea in mucopolysaccharidoses. Mol Genet Metab. 2014;112:154-9.

35. Barker MJ, García C. Broncoaspiración en el niño. Rol diagnóstico de los estudios de imágenes. Neumol Pediatr. 2015;10:82-5.

36. García R, Beltrán C. Aporte del estudio endoscópico de la deglución en niños con limitaciones neurológicas. Neumol Pediatr. 2011;6:80-3.

37. Ponce M, Garrigues V, Ortiz V, Ponce J. Trastornos de la deglución: un reto para el gastroenterólogo. Gastroenterol Hepatol. 2007;30:487-97.

38. Velasco-Vargas KS, Maldonado-Navas C, Medina T. Evaluación fonoaudiológica de la disfagia: encuentros y disonancias. Rev Colomb Rehabil. 2016;15:22-31.

39. Nazar MG, Ortega TA, Fuentealba MI. Evaluación y manejo integral de la disfagia orofaríngea. Rev Med Clin Condes. 2009;20:449-57.

40. Sitton M, Arvedson J, Visotcky A, Braun N, Kerschner J, Tarima S, et al. Fiberoptic endoscopic evaluation of swallowing in children: feeding outcomes related to diagnostic groups and endoscopic findings. Int J Pediatr Otorhinolaryngol. 2011;75:1024-31.

41. Osona-Rodríguez-de-Torres B, Peña-Zarza JA, Figuerola-Mulet J. Complicaciones respiratorias en el niño con trastorno de deglución y/o reflujo gastroesofágico. Protoc Diagn Ter Pediatr. 2017;1:343-56.

42. Serrano M, Vilaseca MA, Campistol J. Errores congénitos del metabolismo y práctica deportiva. Apunts Med Esport. 2010;45:185-9.

43. Martins AM, Dualibi AP, Norato D, Takata ET, Santos ES, Valadares ER, et al. Guidelines for the management of mucopolysaccharidosis type I. J Pediatr. 2009;155:S32-S46.

44. Valencia-Valencia D, Ruiz-Ospina E, Escobar PA. Rehabilitación pulmonar en niños: un reto para los profesionales en medicina física y rehabilitación. Rev Colomb Med Fis Rehab. 2013;23:70-81.

45. Torres-Castro R, Zenteno D, Rodríguez-Núñez I, Villarroel G, Álvarez C Gatica D, et al. Guías de rehabilitación respiratoria en niños con enfermedades respiratorias crónicas: actualización 2016. Neumol Pediatr. 2016;11:114-31.

46. Guía de Práctica Clínica. Cuidados respiratorios en pacientes con mucopolisacaridosis y enfermedad de Pompe. México: Secretaría de Salud; 2013. Disponible en: www.cenetc.salud.gob.mx/interior/gpc.ht/m

47. García-Ramos EC, Santana-Rodríguez I. Fisioterapia respiratoria. Indicaciones y formas de aplicación en el lactante y el niño. An Pediatr Contin. 2011;9:316-9.

48. Martínez-Carrasco C, Cols-Roig M, Salcedo-Posadas A, Sardon-Prado O, Asensio-de-la-Cruz O, Torrent-Vernetta A. Tratamientos respiratorios en la enfermedad neuromuscular. An Pediatr (Barc). 2014;81:259.e1-9.

49. Torres-Castro R, Monge G, Vera R, Puppo H, Céspedes J, Vilaró J. Estrategias para aumentar la eficiencia de la tos en pacientes con enfermedades neuromusculares. Rev Med Chile. 2014;142:238-45. 
50. Postiaux G, Dubois R, Marchand E, Demay M, Jacquy J, Mangiaracina M [Expiration lente prolongée et toux provoquée dans la bronchiolite du nourrisson]. Kinesither Rev. 2006;55:35-41.

51. Barros-Poblete M, Torres-Castro R, Villaseca Y, Ríos C, Puppo H, Rodríguez-Núñez I, et al. Consenso chileno de técnicas de kinesiología respiratoria en pediatría. Neumol Pediatr. 2018;13:137-48.

52. Gómez-Merino E, Sancho J, Marín J, Servera E, Blasco ML, Belda JF, et al. Mechanical insufflation-exsufflation: pressure, volume, and flow relationships and the adequacy of the manufacturer's guidelines. Am Phys Med Rehabil. 2002;81:579-83.

53. Cortes-Télles A, Che-Morales JL, Ortiz-Farías DL. Estrategias actuales en el manejo de las secreciones traqueobronquiales. Neumol Cir Torax. 2019;78:313-23.

54. Garuti G, Lusuardi M, Bach JR. Management of cough ineffectiveness in neuromuscular disorders. Shortness of Breath. 2013:2:28-34.

55. Toussaint M, De Win H, Steens M, Soudon P. Effect of intrapulmonary percussive ventilation on mucus clearance in Duchenne muscular dystrophy patients: a preliminary report. Respir Care. 2003;48:940-7.

56. Fernández-Restrepo L, Shaffer L, Amalakuhan B, Restrepo MI, Peters J, Restrepo R. Effects of intrapulmonary percussive ventilation on airway mucus clearance: a bench model. World J Crit Care Med. 2017;6:164-71.

57. Deakins K, Chatburn RL. A comparison of intrapulmonary percussive ventilation and conventional chest physiotherapy for the treatment of atelectasis in the pediatric patient. Respir Care. 2002;47:1162-7.

58. O'Neill K, O'Donnell AE, Bradley JM. Airway clearance, mucoactive therapies and pulmonary rehabilitation in bronchiectasis. Respirology. 2019;24:227-37.
59. McPeck M. Vibralung acoustical percussor: a new paradigm in airway clearance therapy. Respir Ther. 2014;9:45-7.

60. Chatburn RL. High-frequency assisted airway clearance. Respir Care. 2007;52:1224-35

61. Yuan N, Kane P, Shelton K, Matel J, Becker BC, Moss RB. Safety, tolerability, and efficacy of high-frequency chest wall oscillation in pediatric patients with cerebral palsy and neuromuscular diseases: an exploratory randomized controlled trial. J Child Neurol. 2010;25:815-21.

62. Gómez-Grande ML, González-Bellido V, Olguín G, Rodríguez H. Manejo de las secreciones pulmonares en el paciente crítico. Enferm Intensiva. $2010 ; 21: 74-82$.

63. Paiva DN, Assmann LB, Bordin DF, Gass R, Jost RT, Filho MB, et al Inspiratory muscle training with threshold or incentive spirometry: which is the most effective? Rev Port Pneumol. 2015;21:76-81.

64. Örman J, Westerdahl E. Chest physiotherapy with positive expiratory pressure breathing after abdominal and thoracic surgery: a systematic review. Acta Anaesthesiol Scand. 2010;54:261-7.

65. Fagevik-Olsen M, Lannefors L, Westerdahl E. Positive expiratory pressure: common clinical applications and physiological effects. Respir Med. 2015;109:297-307.

66. Martínez-Carrasco C, Cols-Roig M, Salcedo-Posadas A, Sardón-Prado O, Asensio-de-la-Cruz O, Torrent-Vernetta A. Tratamientos respiratorios en la enfermedad neuromuscular. An Pediatr (Barc). 2014;81:259.e1-9.

67. Daily JC, Wang HE. Noninvasive positive pressure ventilation: resource document for the National Association of EMS physicians. Prehosp Emerg Care. 2011;15:432-8 